\title{
Educación financiera como estrategia de responsabilidad social de instituciones bancarias de Guayaquil
}

\author{
Miguel Cueva Estrada* \\ Jorge Montecel Shiguango** \\ Michelle Viera Romero*** \\ Ecuador
}

\section{Introducción}

Las crisis económicas anteriores y la actual han tenido un alto impacto entre la población generando cierta desconfianza hacia los productos financieros. En razón de esta situación se han creado las herramientas e información necesarias para que las personas puedan tomar mejores decisiones financieras que fomenten el desarrollo y bienestar de sus familias y negocios. Por este motivo, la banca ecuatoriana junto con la del resto del mundo ha valorado la importancia de educación financiera entre sus clientes.

De acuerdo a los antecedentes expuestos se estima importante diagnosticar y evaluar las experiencias de educación financiera que

* Máster en Administración de Empresas. Universidad de Guayaquil. Docente de la Facultad de Ciencias Administrativas. Contacto: nathaelcueva@gmail. com

** Máster en Administración de Empresas. Universidad de Guayaquil. Docente de la Facultad de Ciencias Administrativas. Contacto: jorge.montecelsh@ ug.edu.ec

*** Máster en Administración Pública. Universidad de Guayaquil. Docente de la Facultad de Ciencias Administrativas. Contacto: michelle.vierarom@ug.edu.ec 
han desarrollado distintas instituciones bancarias del sector privado dentro de la ciudad de Guayaquil a fin de contar con elementos de análisis suficientes respecto al cumplimiento de la responsabilidad social y facilitar herramientas a la población para combatir la pobreza y promover la equidad intergeneracional, a pesar de no existir lineamientos aplicables de políticas de educación financiera en Ecuador.

Mediante la recopilación de información mediante fichas técnicas se va a exponer la relación que existe entre la educación financiera avalada por la Superintendencia de Bancos con la Guía de Responsabilidad Social ISO-26000, para luego diagnosticar los programas de educación financiera en instituciones bancarias de la ciudad de Guayaquil.

Por definición, la Responsabilidad Social (RS) es una actitud participativa que nos permite adoptar hábitos, procesos y estrategias que puedan minimizar los impactos negativos que se podrían generar desde el punto de vista económico, medio ambiental y social (ISO, 2010).

La norma Iso-26000 contiene ocho materias fundamentales que contienen diversos asuntos. En el caso de las instituciones bancarias, la educación financiera puede ser incorporada como una estrategia que permita a estas instituciones ser socialmente responsables. Es por esta razón que se buscará determinar en qué asunto de responsabilidad social se encuentra ubicada la educación financiera y a su vez establecer cuál es su estado actual en todas las instituciones bancarias que tienen matriz en la ciudad de Guayaquil (IsO, 2010).

La principal fuente de análisis para el presente estudio fueron las páginas webs de las instituciones de la ciudad de Guayaquil para determinar el estado actual de la educación financiera. Se ha bosquejado una línea base y aportado un insumo para la estandarización de la educación financiera apegada a principios y asuntos de responsabilidad social, obteniendo así los niveles de cumplimiento de las instituciones bancarias de la ciudad. 


\section{Fundamentos teóricos}

\section{Educación financiera y responsabilidad social empresarial en instituciones bancarias}

La responsabilidad social es el compromiso que asume una entidad para contribuir al desarrollo económico sostenible por medio de la cooperación de su entorno interno y externo, como sus empleados, familias, la comunidad local y la sociedad, con el objeto de mejorar la calidad de vida (Iso, 2010).

Una de las máximas guías de responsabilidad social es la Iso-26000 de la cual se tomará los asuntos determinados por esta para determinar con cuál de los asuntos de responsabilidad social está alineada la educación financiera del Ecuador.

La educación financiera es una herramienta fundamental para la inclusión porque no solo facilita el uso efectivo de los productos financieros, sino que también ayuda a que las personas desarrollen las habilidades para comparar y seleccionar los mejores productos para sus necesidades y los empodera para ejercer sus derechos y responsabilidades (CAF, 2013).

Las instituciones bancarias de otros países como Colombia y Perú como parte de su responsabilidad social empresarial utilizan a la educación financiera como estrategia con la finalidad de llegar a la comunidad permitiendo que la sociedad (clientes y comunidad) pueda tomar decisiones informadas mejorando la gestión del riesgo y así poder promover el buen manejo del dinero dentro de un sistema financiero.

\section{Educación financiera en Ecuador}

En el Ecuador la educación financiera nace de la Constitución de la República del Ecuador del año 2008, y con ella todas las normativas derivadas que tratan sobre educación financiera, que nacen específicamente de los artículos 52 y 55 de la Constitución, donde se determina que es deber del Estado garantizar el derecho de los ciudadanos a

disponer de bienes y servicios de óptima calidad; a elegirlos con libertad, así como a recibir información precisa y no engañosa 
sobre su contenido y características; que la ley establecerá los mecanismos de control de calidad, los procedimientos de defensa del consumidor, la reparación e indemnización por deficiencias, daños o mala calidad de bienes y servicios; y, que las personas usuarias y consumidoras podrán constituir asociaciones que promuevan la información y educación sobre sus derechos. (Asamblea Nacional, 2008)

Con base en los artículos de la Constitución y en el Código Orgánico Monetario Financiero (COMF) se establece cuál es el principal mecanismo de control dentro del sistema financiero nacional. Con base en la normativa de esta institución se realizará el diagnóstico de la educación financiera en el Ecuador y en la Ciudad de Guayaquil. Específicamente, el artículo 60 del Código Orgánico Monetario Financiero determina que es la Superintendencia de Bancos la responsable de vigilar, auditar, intervenir, controlar y supervisar actividades financieras que ejercen las entidades públicas y privadas del sistema financiero nacional. Así mismo, el artículo 62 de este mismo Código menciona que la Superintendencia de Bancos podrá expedir las normas en las materias propias de su competencia, sin que puedan alterar o innovar las disposiciones legales ni las regulaciones que expida la junta de política y regulación monetaria financiera. (Asamblea Nacional, 2014).

La educación financiera en el Ecuador también está contemplada en el instrumento máximo de planificación que es el Plan Nacional del Buen Vivir 2013-2017, en específico en su Objetivo 4.9 literal m) “Generar programas y contenidos educativos que difundan y fortalezcan la educación financiera" (Plan Nacional del Buen Vivir, 2009).

Es a partir de ahí que la Junta Bancaria resuelve, a través de la resolución No. JB-2013-2393 del 22 de enero de 2013, disponer que las entidades financieras controladas por la Superintendencia de Bancos desarrollen Programas de Educación Financiera (PEF) en beneficio de sus clientes y público en general. El objetivo final de esta iniciativa es dotar a la población de conocimientos que apoyen la toma de decisiones sobre sus finanzas personales y familiares; seguros y seguridad social, que amplíen sus oportunidades de participación informada y responsable a lo largo de la vida económica de las personas. 


\section{Superintendencia de Bancos del Ecuador}

La Superintendencia de Bancos define a la educación financiera como el proceso mediante el cual la población aprende o mejora la comprensión de los conceptos, características, costos y riesgos de los productos y servicios financieros, así como de los derechos y obligaciones de los usuarios financieros, a través de la información, educación y asesoría objetiva recibida, a fin de desarrollar capacidades que le permitan tomar decisiones financieras y ejercitar sus derechos en condiciones que le proporcionan bienestar personal y familiar.

La resolución SB-2015-665 de la Superintendencia de Bancos regula los programas de Educación Financiera. A través de los principios y objetivos de dicha disposición, se pudo realizar la presente investigación que analizará los parámetros vigentes de responsabilidad social con los programas actuales de Educación Financiera (Superintendencia de Bancos del Ecuador, 2015).

\section{Metodología}

El presente diagnóstico permitirá determinar si la educación financiera es una estrategia de responsabilidad social. Para esta actividad se hizo uso de fuentes secundarias de información como las páginas web de las instituciones financieras a evaluar, resoluciones de la Superintendencia de Bancos y la normativa de Iso-26000. Como unidad de análisis se ha considerado a bancos que poseen matriz en la ciudad de Guayaquil.

La presente investigación realizó dos aportes a la educación financiera, primero determinó a qué asunto de la IsO-26000 se encuentran alineados los objetivos de la Resolución SB-2015-665, y la situación actual de la educación financiera de la ciudad de Guayaquil con respecto a los principios de educación financiera.

Esta evaluación pasó por tres etapas. En la primera etapa se escogieron los objetivos de la Resolución SB-2015-665 y los asuntos de responsabilidad social de las normas ISO-26000, y se elaboró la matriz de cumplimiento para determinar el enlace de la educación financiera con las normas Iso-26000. En la segunda etapa, una vez comprobado el enlace de la educación financiera del país con la Iso-26000, se realizó 
el análisis de las páginas web de los bancos de Guayaquil a estudiar. En la tercera etapa se estimará el nivel de cumplimiento de la educación financiera con base en los principios de la Resolución SB-2015665 de cada uno de los Bancos.

\section{Elementos para la elaboración de las matrices}

\section{Resolución SB-2015-665: objetivos y principios, programas de educación financiera}

Para el respectivo análisis se usaron y analizaron los principios y objetivos de la resolución mencionada que servirán para calcular el nivel de cumplimiento de las normas de educación financiera de instituciones bancarias de Guayaquil.

\section{ISO-26000 Asuntos de responsabilidad social}

La norma Iso-26000 se divide en materias fundamentales con sus respectivos asuntos que fueron tomados para su análisis.

\section{Bancos de la ciudad de Guayaquil objeto de análisis}

Los Bancos que fueron objeto de este estudio fueron: Banco del Pacífico, Banco de Guayaquil, Banco del Litoral, Banco Amazonas, Banco Coop. Nacional, Banco Bolivariano, Banco Dmiro, Banco Del Bank.

\section{Matrices creadas para el estudio}

Con los elementos anteriormente mencionados se procedió a elaborar las respectivas matrices para cada una de las etapas del proceso de evaluación de la banca de la ciudad de Guayaquil.

-Etapa 1 - Matriz 1: Matriz de cumplimiento de la norma ISO26000 en los objetivos de la resolución SB-105-665 
Mediante el uso de los asuntos de responsabilidad social y los objetivos de la Resolución SB-105-665 se pudo construir esta matriz, la cual tiene como finalidad determinar si existe una relación de los objetivos de los programas de educación financiera con los asuntos de la norma IsO-26000.

-Etapa 2 - Matriz 2: Matriz de evaluación de programas de educación financiera en los Bancos de la ciudad de Guayaquil

Una vez analizada la relación existente entre las normas de responsabilidad social y las normas de la educación financiera del país se procedió, mediante la utilización de los principios de los programas de educación financiera del país, a la construcción de la mencionada matriz de evaluación de programas de educación financiera, que tiene como finalidad evaluar el cumplimiento de los programas de educación financiera actual en la ciudad de Guayaquil.

\section{Resultados}

Mediante la construcción de las matrices de evaluación se obtuvieron los siguientes resultados.

- Etapa 1 - Cumplimiento con la Norma IsO-26000

Cabe destacar que todos los objetivos de la educación financiera en el Ecuador ubicados en la normativa SB-2015-665 se encuentran alineados en algún asunto de responsabilidad social, mayormente en la Materia Fundamental de Asuntos de Consumidor en los Asuntos 1, 4 y 7.

- Objetivo 4.1: Se alinea con los asuntos 5 y 7 de la materia fundamental sobre Derechos Humanos y con el Asunto 7 de la materia fundamental de Asuntos del Consumidor.

- Objetivo 4.2: Se alinea el asunto 7 de la materia fundamental sobre Derechos Humanos y con los Asuntos 1 y 7 de la materia fundamental de Asuntos del Consumidor. 
- Objetivo 4.3: Se alinea con los asuntos 1,4 y 7 de la materia fundamental de Asuntos del Consumidor.

- Objetivo 4.4: Se alinea con el asunto 7 de la materia fundamental Derechos Humanos y con los asuntos 1,4 y 7 de la materia fundamental de Asuntos del Consumidor.

- Objetivo 4.5: Se alinea con el asunto 5 de la materia fundamental Derechos Humanos, con los asuntos 1,4 y 7 de la materia fundamental Asuntos del Consumidor y con el Asunto 1 de la materia fundamental de Participación Activa y Desarrollo de la Comunidad.

- Objetivo 4.6: Se alinea con los asunto 1,4 y 7 de la materia fundamental de Asuntos del Consumidor.

- Etapa 2 - Evaluación de los programas de educación financiera de los Bancos de la ciudad de Guayaquil basado en los principios de educación financiera

Dentro de los principales resultados de esta evaluación por indicador cabe destacar que los bancos del Litoral y Dmiro no tienen programas de educación financiera en sus páginas web, y se se obtuvo lo siguiente:

- Accesibilidad: Los bancos del Pacífico, Amazonas, Coop. Nacional y Delbank tienen una accesibilidad satisfactoria al poder ubicarse los links del programa de manera fácil y rápida. Los bancos de Guayaquil y Bolivariano tienen un difícil acceso a sus programas de educación financiera.

- Realización de Promoción: Ninguno de los bancos evaluados promociona sus programas de educación financiera.

- Contenidos: De acuerdo a la temática planteada en los principios de la Superintendencia de Bancos se destaca que las temáticas de mercado de valores y tributos no son tomadas en consideración por ningún banco. Los bancos del Pacífico, Bolivariano y Coop Nacional son los que poseen un mayor grado de cumplimiento de la temática planteada por la Superintendencia de Bancos, con un $78 \%$ de cumplimento de los temas propuestos. Queda como fuente para otra investigación verificar si estos 
contenidos causan algún impacto en el conocimiento de las personas de distintos púbicos.

- Población objetivo: Solo los bancos Coop. Nacional y Bolivariano definen población objetivo por rangos de edades haciendo la diferenciación de los productos y servicios para cada segmento.

- Periodicidad: Ningún banco de la ciudad de Guayaquil define qué tiempo duran sus programas de educación financiera, lo que significa que la temática planteada solo es esquematizada en los portales.

- Sensibilización sobre problemas financieros: Los bancos Amazonas, Pacífico y Coop. Nacional mencionan en sus programas los problemas financieros latentes en el país.

- Diferenciación de asesoría comercial y promoción de productos y servicios: Todos los bancos de la ciudad de Guayaquil tienen esa diferenciación.

- Identificación de grupos de interés: No existe una identificación de stakeholders para los programas de educación financiera.

- Cooperación con otros programas: Solo el Banco Bolivariano posee alianza con otras instituciones para ofrecer educación financiera.

- Metodología clara y objetiva: Los bancos Pacífico, Coop. Nacional y Amazonas poseen una metodología esquematizada con objetivos planteados.

- Calculadora financiera: Solo el Banco Del Bank no posee una calculadora financiera.

- Etapa 3 - Nivel de Cumplimiento Instituciones Bancarias de la Ciudad de Guayaquil

La investigación en primera instancia verificó si la educación financiera en el Ecuador es una estrategia enfocada en la responsabilidad social, para lo cual se enlazó los objetivos de la Superintendencia de Bancos con las normas Iso-26000, lo cual se consideró que si se asocia con los asuntos de responsabilidad social, mayormente en la materia fundamental de Asuntos del Consumidor.

Una vez comprobada que efectivamente la educación financiera en el país está enlazada a la responsabilidad social, se procedió mediante 
los principios a evaluar los programas de educación financiera en la unidad de análisis para lo cual se recurrió a las páginas web de las instituciones mencionadas, obteniendo una matriz de observación que permitió la evaluación. Los resultados consolidados en esta medición dieron como resultado el nivel de cumplimiento de los principios de los programas de educación financiera de las instituciones bancarias de la ciudad de Guayaquil (ver cuadro 1).

Cuadro 1. Nivel de cumplimiento de principios de programas de educación financiera en instituciones bancarias de Guayaquil

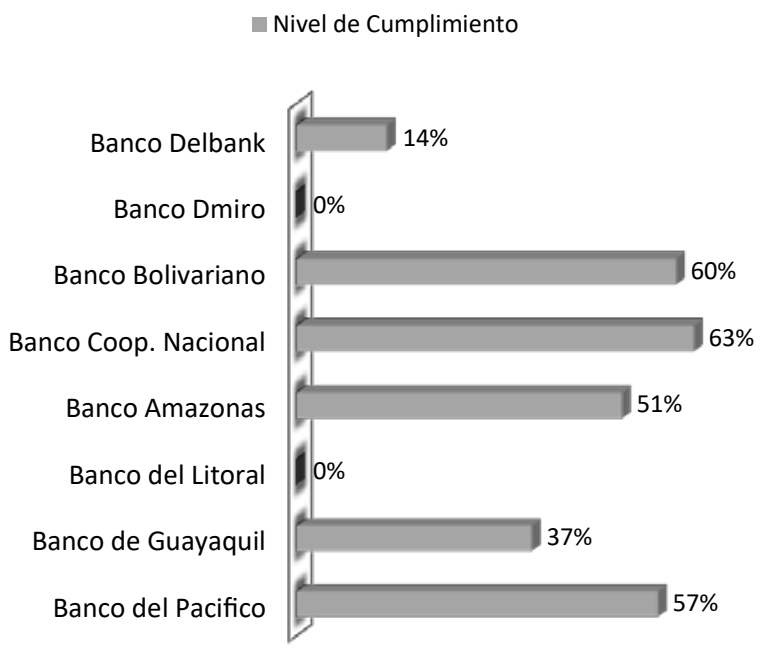

Fuente: elaboración propia.

\section{Discusión}

Solo se realizó la evaluación de las instituciones bancarias de la ciudad de Guayaquil, por lo que aquellas instituciones que no poseen su agencia matriz en la ciudad no fueron tomadas en cuenta en esta evaluación por lo que los resultados de la primera etapa se podrían aplicar a todas las instituciones bancarias del Ecuador.

La Superintendencia de Bancos regional con sede en Guayaquil por lo general realiza evaluaciones en mencionadas instituciones. Esta 
evaluación se la realiza mediante metas, por lo que las instituciones bancarias están en la obligación de realizar mejoras en sus interfaces.

1. La Normativa de la Superintendencia de Bancos podría cambiar, por lo que la matriz de diagnóstico realizada puede sufrir modificaciones en su estructura pero la medición que se sugiere en esta investigación es la que más se apega a la normativa vigente.

2. Si bien es cierto que existe cumplimiento por parte de las instituciones otro punto a analizar para una próxima ocasión es la calidad de lo ofertado en los programas de educación financiera.

3. El nivel de cumplimiento mayor de las entidades bancarias pertenece al Banco Coop. Nacional con el $63 \%$.

4. Banco Dmiro y el Banco del Litoral no posee una plataforma o sección de Educación Financiera, por lo que poseen un nivel de $0 \%$ de Cumplimiento.

Ningún banco de Guayaquil en sus plataformas y sección de Educación Financiera posee los temas de tributos y mercados de valores provocando el desconocimiento en estos temas comprobando además la poca participación ciudadana en el mercado de valores.

Ningún banco de la ciudad reconoce abiertamente a sus grupos de interés, lo que genera que no se conozca el entorno de las entidades bancarias.

\section{Conclusiones}

Luego del contraste entre la ISO-26000 y la resolución SB-2015-665 se evidencia como conclusiones que:

La educación financiera del Ecuador se encuentra asociada a principios de responsabilidad social en temas de Derechos Humanos y Asuntos de Consumidor cumpliendo con los aspectos amparados en la Constitución del Ecuador, por lo que se considera que la educación financiera en el país es socialmente responsable. 
La educación financiera en el país puede ser usada por las diversas entidades financieras como estrategia de responsabilidad social empresarial del sector.

La Superintendencia de Bancos del Ecuador es la entidad que en la actualidad posee los mecanismos de control necesarios como reglamentos y normativas para realizar el control de las instituciones del país.

A los bancos de la ciudad de Guayaquil aún le faltan aspectos básicos de una estructura de un programa de educación financiera, lo que deriva en que los mencionados programas no cumplan en su totalidad los parámetros solicitados por la Superintendencia de Bancos del Ecuador.

Finalmente, la responsabilidad social empresarial en el sector financiero se ve reflejada en la educación financiera, cuyos aspectos en el Ecuador aún se muestran en una etapa de crecimiento; al respecto, no se han definido los grupos de interés y no se han formado alianzas entre universidades y la Superintendencia de Bancos. Con el desarrollo de una cultura de educación financiera se podría eliminar el desconocimiento y desconfianza sobre productos y servicios financieros.

Para futuras investigaciones, sería de interés estudiar el impacto de las herramientas actuales hacia la sociedad o clientela del sector financiero en la ciudad de Guayaquil, lo que permitiría demostrar si estas herramientas fomentan el crecimiento de la sociedad guayaquileña. 


\section{Referencias}

Asamblea Nacional. (2008). Asamblea Nacional. Obtenido de Gaceta Oficial: http://www.asambleanacional.gov.ec/documentos/constitucion_de_ bolsillo.pdf

Asamblea Nacional. (2014). Asamblea Nacional. Obtenido de Registro Oficial: http://www.asambleanacional.gob.ec/es/system/files/ro_codigo_organico_monetario_y_financiero.pdf

Bansefi. (2014). Bansefi. Obtenido de Bansefi: http://www.bansefi.gob.mx/ Pages/Inicio.aspx

CAF. (2013). CAF. Obtenido de Scioteca: http://scioteca.caf.com/ handle/123456789/379

Coates, K. (2009). Educación financiera: temas y desafís para América Latina. Conferencia Internacional OCDE-Brasil sobre Educación financiera. [Diapositivas de PowerPoint]. Organisation for Economic Co-Operation and Development. Conferencia llevada a cabo en Río de Janeiro, Brasil. Recuperado de http://www.oecd.org/finance/financial-education/44264471.pdf

Congreso Nacional. (2000). Ley Orgánica de Defensa del Consumidor. Publicado en Registro Oficial Suplemento No. 116 del 10 de julio de 2000. Última modificación 2011. Recuperado de http://www.industrias.gob. ec/wp-content/uploads/2015/04/A2-LEY-ORGANICA-DE-DEFENSA-DEL-CONSUMIDOR.pdf

Falçao Martins, H. \& Fontes Filho, J. (1999). ¿En quién se pone el foco? Identificando stakebolders para la formulación de la misión organizacional. Revista del CLAD Reforma y Democracia, No. 15 (Oct. 1999). Caracas. Recuperado de http://siare.clad.org/revistas/0033942.pdf

García, N. Grifoni, A. \& López, J. (2013). La educación financiera en América Latina y el Caribe. Corporación Andina de Fomento. Recuperado de https://www.oecd.org/daf/fin/financial-education/OECD_CAF_Financial_Education_Latin_AmericaES.pdf

ISO. (2010). ISO-26000. Geneva-Suiza: ISO ORG.

Junta Bancaria. (2013). Resolución No. JB-2013-2393 del 22 de enero de 2013. Recuperado de http://www.sbs.gob.ec/medios/PORTALDOCS/ downloads/normativa/nueva_codificacion/todos/L1_XIV_cap_IV.pdf

Mitchell, R. Agle, B. and Wood, D. (1997). Toward a Theory of Stakeholder Identification and Salience: Defining the Principle of Who and What Really Counts. The Academy of Management Review, Vol 22, No. 4 (Oct. 
1997). pp. 853-886. Recuperado de http://courses.washington.edu/ilis580/ readings/Mitchell_et_al_1997.pdf

OECD. (2013). Financial Education . Obtenido de OECD ORG: https://www. oecd.org/daf/fin/financial-education/OECD_CAF_Financial_Education_ Latin_AmericaES.pdf

Secretaría Nacional de Planificación y Desarrollo - Senplades. (2013). Plan Nacional de Desarrollo / Plan Nacional para el Buen Vivir 2013-2017. Recuperado de http://documentos.senplades.gob.ec/Plan\%20Nacional\%20Buen\%20Vivir\%202013-2017.pdf

Superfinanciera. (2010). Superfinanciera. Obtenido de https://www.superfinanciera.gov.co/SFCant/ConsumidorFinanciero/estratenaledufinanciera012011.pdf

Superintendencia de Bancos y Seguros (2015). Resolución No. SB-2015-665 del 17 de agosto de 2015. Recuperado de http://www.bde.fin.ec/sites/ default/files/Resolucio\%CC\%81n\%20SB-2015-665\%20de\%2017\%20 de\%20agsoto \%20de\%202015.pdf

Superintendencia de Bancos del Ecuador. (2009). Preguntas Frecuentes. Generalidades del Sistema Financiero - ¿Quiénes integran el Sistema Financiero Ecuatoriano? Recuperado de http://www.superbancos.gob.ec/practg/ sbs_index?vp_art_id=1\&vp_tip=11\&vp_lang=1\&vp_buscr=11\#131

Superintendencia de Bancos y Seguros del Ecuador. (2015). Superintendencia de Bancos. Obtenido de Resoluciones: http://www.superbancos.gob.ec/ practg/sbs_index?vp_art_id=755\&vp_tip=2

Weimer, D. \& Vining, A. (1989). Policy Analysis. Concepts and practices. New Jersey: Prentice Hall. 